Canadian

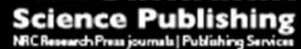

Canadian Journal of Physiology and Pharmacology Revue canadienne de physiologie et pharmacologie

\title{
Age-related testosterone declines can be detected in men's fingernails
}

\begin{tabular}{|r|l|}
\hline Journal: & Canadian Journal of Physiology and Pharmacology \\
\hline Manuscript ID & cjpp-2017-0193.R1 \\
\hline Manuscript Type: & Article \\
\hline Date Submitted by the Author: & 02-May-2017 \\
\hline $\begin{array}{r}\text { Complete List of Authors: } \\
\text { Is the invited manuscript for } \\
\text { consideration in a Special } \\
\text { Issue?: }\end{array}$ & N/A \\
\hline Keyword: Lee; Bar Ilan University, \\
\hline & $\begin{array}{l}\text { biomonitoring, endogenous testosterone levels, fingernails, integrated } \\
\text { matrices, non-invasive }\end{array}$ \\
\hline
\end{tabular}




\title{
Age-related testosterone declines can be detected in men's fingernails
}

\author{
Devorah Matas and Lee Koren ${ }^{\#}$ \\ The Mina and Everard Goodman Faculty of Life Sciences \\ Bar-Ilan University \\ Ramat Gan, 5290002 \\ Israel
}

Corresponding author:

${ }^{\#}$ Lee Koren, Ph.D.

The Mina and Everard Goodman Faculty of Life Sciences,

Bar Ilan University, Ramat Gan, Israel;

Phone: +972-3-7384371

Fax: $+972-3-7384372$

E-mail: Lee.Koren@biu.ac.il

Devorah Matas, Ph.D.

The Mina and Everard Goodman Faculty of Life Sciences,

Bar Ilan University, Ramat Gan, Israel;

Phone: $+972-3-7384372$

E-mail: Devorahbio@gmail.com 


\begin{abstract}
Testosterone plays multiple roles in the regulation of development, physiology, reproduction, and behavior. Age-related testosterone declines are expected in the population. However, measuring circulating testosterone is especially challenging since concentrations are labile, responding to social situations and challenges. Matrices that integrate long-term testosterone levels are therefore valuable as biomarkers of mean, as well as chronic exposures. Here we report on a simple method to extract and measure accumulated testosterone from human fingernails using commercial EIA kits. Further, we demonstrate known human testosterone sex and age trends. Our method is especially useful for quantifying testosterone in men's nails, where a small amount of matrix is required. Thus, this approach is a potential tool for biomonitoring endogenous as well as exogenous testosterone exposure. We suggest considering nails as an alternative matrix for quantifying other steroids as well.
\end{abstract}

Key words: biomonitoring, endogenous testosterone levels, fingernails, integrated matrices, non-invasive. 


\section{Introduction}

Steroid hormones influence and are influenced by development, physiology, and behavior. Circulating and integrated steroid hormone levels, such as glucocorticoids and androgens, can provide information on growth and reproduction, and offer prospective biomarkers for wellbeing and survival (Koren et al. 2012). Measuring these steroids in integrated matrices, such as hair, feathers, and nails, is a progressively developing field (Gosetti et al. 2013; Koren et al. 2002; Koren et al. 2012). Unlike traditional blood sampling, hair and nail collection is relatively non-invasive, pain and infection-free. Hair and nails may be cut and collected quickly and efficiently, without the need for patient privacy or researcher health precautions. They are also easy to transport and store, without needing electricity and cooling. Moreover, while circulating steroid hormone levels represent the momentary total (i.e., protein-bound and free) state, hair and nails provide a measure of the average level of bioavailable free steroids over weeks or months (i.e., time of their growth) (Palmeri et al. 2000).

In mammals, nails develop by keratinocyte cell divisions in the proximal germinal regions (Baran 1981; De Berker et al. 2007). Nail growth is attributed primarily to the nail matrix and to a lesser extent to the nail bed. The nail matrix is mainly composed of specific keratin proteins, cross-linked by keratin-associated proteins. Diverse factors influence nail growth, including numerous physiological and pathological conditions (reviewed in Zaiac \& Walker (Zaiac and Walker 2013)). On average, human fingernails grow at a rate of $3 \mathrm{~mm}$ per month, while human toenails grow at a rate of $1 \mathrm{~mm}$ per month. During nail growth, multiple materials are transferred from the nail bed and matrix to the growing nail (reviewed in (Palmeri et al. 2000)). These are embedded in the growing nails, making this abundant matrix an attractive candidate for biomonitoring multiple blood borne substances. Indeed, poisonous materials (Barbosa et al. 2005), drugs (e.g. (Shu et al. 2015)) and endocrine-disruptors (Li et al. 2013) have been forensically detected in human nails. In addition, endogenous steroid hormones have been analyzed in nail extracts. For example, infants that experienced in utero stress had higher DHEA (dehydroepiandrosterone) in their nails compared to infants from 
non-stressed mothers (Tegethoff et al. 2011). In another study, nail cortisol-to-DHEA ratio was found to be related to perceived exam stress in students (Warnock et al. 2010).

Exogenous testosterone and its derivatives were measured in nails using LC-MS (Brown and Perrett 2011). However, the authors concluded that detection of these substances was not sensitive enough. GC-MS was successfully used in a different study, following the required derivatization (Choi et al. 2001). Here, we developed a simple and repeatable quantitative method for measuring endogenous testosterone in men's nails, and assessed its suitability by biologically validating it using well-known age-related trends.

\section{Materials and methods}

\section{Nail collection}

In order to collect nail samples, we sent out an electronic mail in November 2014 requesting fingernail donations from graduate students, staff, and faculty members in the Faculty of Life Sciences at Bar Ilan University. Donated samples were placed in paper envelopes marking only the sex and age of the contributor on it. Envelopes were anonymously deposited in our laboratory's mailbox. By May 2015 we collected 80 nail samples: 52 from men (ages 18.574), 6 from boys (ages 0.4-2), 11 from women (ages 18-53) and 11 from girls (ages 3-9). The study was approved by Bar Ilan University's Research on Human Subjects' Ethics Committee.

\section{Testosterone extraction}

We extracted testosterone from nails using our published protocol for hair and claw-testing (Koren et al. 2002; Koren et al. 2012; Matas et al. 2016), with several modifications. Nails were weighed to the nearest $0.01 \mathrm{mg}$ in safe-lock polypropylene tubes (Sarstedt, Germany). Samples were ground in mixer mill MM 400 (Retsch, Germany) for 10 minutes at $25 \mathrm{~Hz}$. Methanol (UPLC grade, Sigma, Israel) was added, and the minced samples were sonicated for 
30 minutes and then incubated overnight at $50^{\circ} \mathrm{C}$, with gentle shaking. Next day, the supernatant was centrifuged at 17,000 g, transferred into a glass tube (Corning Inc., USA) and evaporated under a stream of nitrogen. Samples were reconstituted and testosterone was quantified in the assay buffer that was provided with the commercial enzyme-linked immunosorbent assays (ELISA) according to manufacturer's recommendations.

\section{Quantitation of testosterone}

High sensitivity salivary testosterone ELISA kits (Salimetrics; item no.1-2402; Ann Arbor, USA) were validated for nails by testing parallelism with kit standards and linearity. Linearity was demonstrated for men between 1-20 mg of nail extract, corresponding to $6-230 \mathrm{pg} / \mathrm{mL}$ testosterone. For women, linearity was observed between 30-50 mg nails, corresponding to $100-200 \mathrm{pg} / \mathrm{mL}$ testosterone. In children, serial dilutions were linear in the $10-50 \mathrm{mg}$ nail extract range, which is equivalent to $38-210 \mathrm{pg} / \mathrm{mL}$ testosterone standards. The lowest concentration that we detected using the assay was $0.41 \mathrm{pg} / \mathrm{mL}$, which was measured in a 3.95 mg boy nail matrix. The antibody used in the commercial ELISA kit is highly specific for testosterone. According to the manufacturer, antibody cross-reactivity is $36.4 \%$ with dihydrotestosterone, $21.02 \%$ with 19 -nortestosterone, $1.9 \%$ with 11 -hydroxytestosterone, $1.157 \%$ with androstenedione and less than $0.489 \%$ for all other steroids. By measuring six replicate pool samples of men nail extract on the same ELISA plate, we calculated intra-assay variability to be $2.7 \%$. By quantifying four samples of the same pool on four different plates and days we calculated inter-assay variability to be $11.78 \%$. Recovery was calculated to be $86 \%$ by the addition of a known amount of testosterone standard to the pool of nail extract. The presence of testosterone in human fingernails was further validated utilizing a second antibody, by a second kit (DRG International Inc. item no. EIA-1559; NJ, USA) following the manufacturer's protocol. DRG reports that the testosterone antibody cross-reacts with $11 \beta$ -

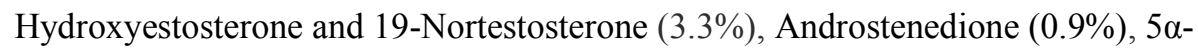
Dihydrotestosterone $(0.8 \%)$ and other steroids $(\leqslant 0.1 \%)$. 


\section{LC-MS/MS validation}

The presence of testosterone in our pooled nails extract was confirmed by LC-MS/MS. Extracted nail samples were dissolved in methanol, spiked with internal standards, and filtered through $0.2 \mu \mathrm{m}$ PVDF filter. Samples were analyzed on a system which consisted of Dionex Ultimate 3000 RS HPLC coupled to Q Exactive Plus hybrid FT mass spectrometer equipped with APCI ion source (Thermo Fisher Scientific Inc.). HPLC separations were carried out using Acclaim C18 column $(2.1 \times 150 \mathrm{~mm}$, particle size $2.2 \mu \mathrm{m}$, Dionex $)$ employing linear water/methanol gradient. Mass spectrometer was operated in positive ionization mode, ion source parameters were as follows: corona discharge needle $5 \mu \mathrm{A}$, capillary temperature $300^{\circ} \mathrm{C}$, sheath gas rate (arb) 50, and auxiliary gas rate (arb) 10 . Mass spectra were acquired in the Parallel Reaction Monitoring mode, and resolving power was set to 70.000 . The system control and data analysis was executed by the Xcalibur software (Thermo Fisher Scientific Inc.).

\section{Statistical analysis}

In order to perform statistical comparisons, testosterone levels were transformed using the Johnson Su transformation to achieve normal distribution. Analysis of variance on the age and sex classes was followed by Tukey-Kramer HSD post-hoc comparisons. Following visualization of the relationship between age and nail testosterone levels, we used non-linear modeling. All statistical tests were performed in JMP 12 (SAS Institute Inc.).

\section{Results}

In order to examine the use of nails as a potential matrix for testosterone quantitation, we conducted validation experiments. Serial dilutions of separate nail pools, each consisting of $>15$ samples, all showed linearity and parallelism with the provided kit standards (univariate analysis of variance; $\mathrm{P}=0.5 ; \mathrm{P}=0.46$ and $\mathrm{P}=0.48$ for men, women, and children respectively; Fig. 1). According to our validations, only $2.5 \mathrm{mg}$ of nails were needed to quantify 
testosterone in males. Ten-fold was necessary to quantify testosterone in females (i.e., women and girls; $25 \mathrm{mg}$ ), comparable to the documented association between circulating testosterone in men vs. women (Longcope 1986). Since a large amount of nail matrix was needed for the female analysis, we created a pool from all donated female samples. We biologically validated our assay by comparing testosterone results for men, women, boys and girls (Fig. 2). Mean testosterone levels in adult men were $5.04 \mathrm{pg} / \mathrm{mg}$ nails ( $\mathrm{N}=52)$, adult women $2.3 \mathrm{pg} / \mathrm{mg}$ nails (a pool of $\mathrm{N}=11$ ), boys $1.8 \mathrm{pg} / \mathrm{mg}$ nails $(\mathrm{N}=6$ ), and girls $1.07 \mathrm{pg} / \mathrm{mg}$ nails (a pool of $\mathrm{N}=11$ ). Analysis of variance showed significant differences in testosterone levels between sex and age groups $\left(\mathrm{F}_{59,3}=9.665 ; \mathrm{P}<0.0001\right)$. Tukey-Kramer post-hoc statistics $(\mathrm{q}=2.648 ; \alpha=0.05)$ showed significant differences in nail testosterone levels between men and boys (difference $1.758 ; \mathrm{P}<0.0001)$. We could not include women and girls in this analysis since we only had a single pooled sample for each. We also examined the changes in testosterone levels as a function of age in males. Over $40 \%$ of the individual variation in male testosterone was due to age, described by a significant quadratic association $\left(\mathrm{R}^{2}=0.405 ; \mathrm{N}=58 ; \mathrm{P}<0.0001 ;\right.$ Fig. 3$)$. This association (inverted-u shape) is a known pattern, and provides further biological validation of our methodology.

\section{Discussion}

Our study shows that nails are an appropriate matrix for quantifying the decline of circulating testosterone in men. We used a simple protocol and commercial ELISA kits to biologically validate men's nails, reflecting well-known sex and age trends. For example, circulating testosterone concentrations are on average higher in men than in women, and higher in adults than in children (Andersson et al. 1997; Mouritsen et al. 2014). Interestingly, in our analysis, we found a 2.5-fold difference between men and women's testosterone levels. Established normal ranges of circulating testosterone concentrations indicate a 7-fold difference between mean concentrations in men and women (Torjesen and Sandnes 2004). The discrepancy between the matrices may reflect the different characteristics of the two. Whereas blood samples reflect a momentary short-lived total (i.e., bound and unbound) testosterone 
concentration, nails presumably show long-term integrated levels of free (i.e., unbound) testosterone, accumulated over weeks. Overall, comparisons of the concentrations of multiple materials in plasma vs. nails show consistently lower results in nails (e.g. (Uematsu et al. 1989)). This may be attributed to the lack of melanin in nails, which reduces material incorporation efficiency (Uematsu et al. 1989). Nails grow both in length and in thickness (Palmeri et al. 2000). These two directions account for distribution of drugs along the entire nail (reviewed in (Palmeri et al. 2000)). However, the detailed mechanism for both endogenous and exogenous material integration from the blood stream into nails requires further investigation. Nonetheless, we needed ten times more nail matrix to quantify testosterone in women. It is also possible that due to the wide variation in testosterone concentrations across individuals, our small sample size for women may have caused an analysis bias. Quantifying testosterone in nails from women and children require further development of our method, in order to increase extraction efficiency that will allow us to use lower nail quantities.

Using miniscule amounts of men's nails, we found a known relationship between age and testosterone levels. Maximal testosterone levels were seen in men that were around 20 years old, and thereafter testosterone gradually declined with age. The non-linear (i.e., inverted-u shaped) association between age and circulating testosterone concentrations has been documented in the literature (Harman et al. 2001). Although individual variation in testosterone is high, and the sample sizes that we obtained of young and old men were low, the emerging pattern was profound. Our study therefore supports the use of nails for comparatively analyzing men's testosterone levels, adding to the body of literature that demonstrates that nails integrate exogenous (e.g. (Shu et al. 2015)) as well as endogenous (Brown and Perrett 2011; Choi et al. 2001; Tegethoff et al. 2011; Warnock et al. 2010) substances. In the future, we would like to further validate nail-testing by comparing testosterone levels in blood, saliva, hair, and nails from the same subjects. In addition, since the analysis of nail testosterone measures the testosterone that was embedded in the keratin matrix at the time of nail formation, in order to establish a more accurate time line, the 
distance of the clipped nail matrix from the root of the nail (i.e., nail length), and the width of the clipping must be taken into account. Therefore, the age of the men that was actually sampled in our study is several months younger than the one that we reported. For further establishment of the protocol for periodic quantitation, repeated nail samples can be cut from the same individual. However, unlike hair that can be cut near the root and segmented to determine the time of hormone deposition, in nails it is more challenging.

In this study, we used commercial, highly sensitive EIAs to quantify testosterone in individual samples. In addition, we unambiguously verified the presence of testosterone in pools of nail extracts using LC-MS/MS. However, we cannot rule out the possibility of testosterone being present as fatty acid esters, or in other forms. Although mass spectrometry is superior in terms of specificity and flexibility to measure multiple compounds simultaneously, a large amount of tissue is initially needed, due to loss during sample prep and cleanup. For example, Choi et al. (2001) used $100 \mathrm{mg}$ of nail matrix to find differences between testosterone in men and women using GC-MS (Choi et al. 2001), and Brown and Perrett (2011) did not find differences between males and females or exogenous testosterone in users and non-users (Brown and Perrett 2011). Here we present a simple and reliable antibody-based method for testosterone quantitation using a small amount of nail matrix, without the need of derivatization or costly equipment. Nail collection is relatively noninvasive, and samples are easy to store and safe to handle. However, it should be considered that nail growth is influenced by age, malnutrition, disease, climate conditions and nail-biting (De Berker et al. 2007). Our method offers the possibility for researchers to quantify testosterone in nails in a simple, applicable way, and document stable long-term trends. For example, it may be useful for identifying changes in testosterone over months for diagnosing various diseases, as well as assessing therapy outcomes (Pastuszak et al. 2016). Further development of a method for measuring testosterone in women's nails will aid diagnosing and treating polycystic ovarian disease (Ehrmann et al. 1995) and hypoandrogenism. Nail-testing also enables periodic quantitation by repeated cutting, opening an exciting array of questions 
and comparative studies examining individual differences in endogenous testosterone levels, as well as exposure to exogenous androgens.

\section{Acknowledgements}

We would like to thank Efrat Koresh for helping to collect nail samples, and to the Bar-Ilan University Faculty of Life Sciences' community for contributing their nails to this study. We also wish to thank Noa Assaf and Shiran Cohen for their help processing the samples, and to Dr. Julius Ben-Ari from the laboratory for the mass spectrometry and chromatography at the interdepartmental analytical unit at The Robert H. Smith Faculty of Agriculture, Food and Environment at the Hebrew University of Jerusalem, Rehovot, for confirming the presence of testosterone using LC-MS/MS.

Conflict of interest: The authors report no conflicts of interest. The authors alone are responsible for the content and writing of the paper.

Role of funding source: none

\section{References}

Andersson, A.M., Juul, A., Petersen, J.H., Muller, J., Groome, N.P., and Skakkebaek, N.E. 1997. Serum inhibin B in healthy pubertal and adolescent boys: relation to age, stage of puberty, and follicle-stimulating hormone, luteinizing hormone, testosterone, and estradiol levels. J. Clin. Endocrinol. Metab. 82(12): 3976-3981. doi: 10.1210/jcem.82.12.4449.

Baran, R. 1981. Nail growth direction revisited. Why do nails grow out instead of up? J. Am. Acad. Dermatol. 4(1): 78-84.

Barbosa, F., Tanus-Santos, J.E., Gerlach, R.F., and Parsons, P.J. 2005. A critical review of biomarkers used for monitoring human exposure to lead: Advantages, limitations, and future needs. Environ. Health Perspect. 113(12): 1669-1674. 
Brown, H.G., and Perrett, D. 2011. Detection of doping in sport: detecting anabolicandrogenic steroids in human fingernail clippings. The Medico-legal journal. 79(Pt 2): 67-69. doi: $10.1258 / \mathrm{mlj} .2011 .011013$.

Choi, M.H., Yoo, Y.S., and Chung, B.C. 20 .01Measurement of testosterone and pregnenolone in nails using gas chromatography-mass spectrometry. J Chromatogr B. 754(2): 495-501.

De Berker, D.A., Andre, J., and Baran, R. 2007. Nail biology and nail science. Int J Cosmet Sci. 241-275:(4)29 doi: 10.1111/j.1467-2494.2007.00372.x.

Ehrmann, D.A., Barnes, R.B., and Rosenfield, R.L. 1995. Polycystic ovary syndrome as a form of functional ovarian hyperandrogenism due to dysregulation of androgen secretion. Endocr. Rev. 16(3): 322-353 .doi: 10.1210/edrv-16-3-322.

Gosetti, F., Mazzucco, E., Gennaro, M.C., and Marengo, E. 2013. Ultra high performance liquid chromatography tandem mass spectrometry determination and profiling of prohibited steroids in human biological matrices. A review .J Chromatogr B, Analytical technologies in the biomedical and life sciences 927: 22-36. doi: 10.1016/j.jchromb.2012.12.003.

Harman, S.M., Metter, E.J., Tobin, J.D., Pearson, J., Blackman, M.R., and Baltimore Longitudinal Study of, A. 2001. Longitudinal effects of aging on serum total and free testosterone levels in healthy men. Baltimore Longitudinal Study of Aging. J. Clin. Endocrinol. Metab. 86(2): 724-731. doi: 10.1210/jcem.86.2.7219.

Koren, L., Mokady, O., Karaskov, T., Klein, J., Koren, G., and Geffen, E. 2002. A novel method using hair for determining hormonal levels in wildlife. Horm and Behav 63: 403-406. Koren, L., Nakagawa, S., Burke, T., Soma, K.K., Wynne-Edwards, K.E., and Geffen, E. 2012. Non-breeding feather concentrations of testosterone, corticosterone and cortisol are associated with subsequent survival in wild house sparrows. Proc. R Soc. Lond. B Biol. Sci. 279(1733): 1560-1566. doi: 10.1098/rspb.2011.2062.

Li, J.G., Guo, F.F., Wang, Y.X., Zhang, J.L., Zhong, Y.X., Zhao, Y.F., and Wu, Y.N. 2013. Can nail, hair and urine be used for biomonitoring of human exposure to perfluorooctane sulfonate and perfluorooctanoic acid? Environ. Int. 53: 47-52. 
Longcope, C. 1986. Adrenal and gonadal androgen secretion in normal females. Clin. Endocrinol. Metab. 15(2): 213-228.

Matas, D., Keren-Rotem, T., and Koren, L. 2016. A method to determine integrated steroid levels in wildlife claws. Gen. Comp. Endocrinol. 230-231: 26-28. doi :

/10.1016j.ygcen.2016.03.020.

Mouritsen, A., Soeborg, T., Johannsen, T.H., Aksglaede, L., Sorensen, K., Hagen, C.P., Mieritz, M.G., Frederiksen, H., Andersson, A.M., and Juul, A. 2014. Longitudinal changes in circulating testosterone levels determined by LC-MS/MS and by a commercially available radioimmunoassay in healthy girls and boys during the pubertal transition. Horm. Res. Paediatr. 82(1): 12-17. doi: 10.1159/000358560.

Palmeri, A., Pichini, S., Pacifici, R., Zuccaro, P., and Lopez, A. 2 .000Drugs in nails: physiology, pharmacokinetics and forensic toxicology. Clin. Pharmacokinet. 38(2): 95-110. doi: 10.2165/00003088-200038020-00001.

Pastuszak, A.W., Rodriguez, K.M., Nguyen, T.M., and Khera, M. 2016. Testosterone therapy and prostate cancer. Transl. Androl. Urol. 5(6): 909-920. doi: 10.21037/tau.2016.08.17.

Shu, I., Jones, J., Jones, M., Lewis, D., and Negrusz, A. 2015. Detection of Drugs in Nails: Three Year Experience. J. Anal. Toxicol. 39(8): 624-628. doi: 10.1093/jat/bkv067.

Tegethoff, M., Raul, J.S., Jamey, C., Khelil, M.B., Ludes, B., and Meinlschmidt, G. 2011. Dehydroepiandrosterone in nails of infants: a potential biomarker of intrauterine responses to maternal stress. Biol. Psychol. 87(3): 414-420. doi: 10.1016/j.biopsycho.2011.05.007. Torjesen, P.A., and Sandnes, L. 2004. Serum testosterone in women as measured by an automated immunoassay and a RIA. Clin. Chem. 50(3): 678; author reply 678-679. doi: 10.1373/clinchem.2003.027565.

Uematsu, T., Sato, R., Suzuki, K., Yamaguchi, S., and Nakashima, M. 1989. Human Scalp Hair as Evidence of Individual Dosage History of Haloperidol - Method and Retrospective Study. Eur. J Clin. Pharmacol. 37(3): 239-244. 
Warnock, F., McElwee, K., Seo, R.J., McIsaac, S., Seim, D., Ramirez-Aponte ,T., Macritchie, K.A., and Young, A.H. 2010. Measuring cortisol and DHEA in fingernails: a pilot study. Neuropsychiatr. Dis. Treat. 6: 1-7.

Zaiac, M.N., and Walker, A. 2013. Nail abnormalities associated with systemic pathologies.

Clin. dermatol. 31(5): 627-649. doi: 10.1016/j.clindermatol.2013.06.018. 


\section{Figure legend}

Figure 1: Validation of nail matrix showing linearity and parallelism of testosterone and provided kit standards. A. Standards vs. men nail samples $(0.5-30 \mathrm{mg})$. B. Standards vs. women nail samples (10-50 mg). C. Standards vs. children nail samples (10-50 mg).

Figure 2: Nail testosterone levels in men, women and children. Testosterone was extracted from $5 \mathrm{mg}$ of nail clippings of individual men $(\mathrm{N}=52)$ and boys $(\mathrm{N}=6)$. Error bars were constructed using 1 standard error from the mean. For women $(\mathrm{N}=11)$ and girls $(\mathrm{N}=11)$ testosterone was extracted from $50 \mathrm{mg}$ of pooled nails. Significant differences were seen between sex and age classes (ANOVA: $\mathrm{F}_{59,3}=9.665 ; \mathrm{P}<0.0001$ ).

Figure 3: Transformed nail testosterone as a function of male age. Transformed testosterone $=$ $-0.151+0.0245 *$ age $-0.0016 *(\text { age- } 28.06)^{2}$. Johnson Su transformation was used to normalize the distribution of testosterone. Polynomial second degree fit $\mathrm{R}^{2}=0.405 ; \mathrm{N}=58$; $\mathrm{P}<0.0001$. 


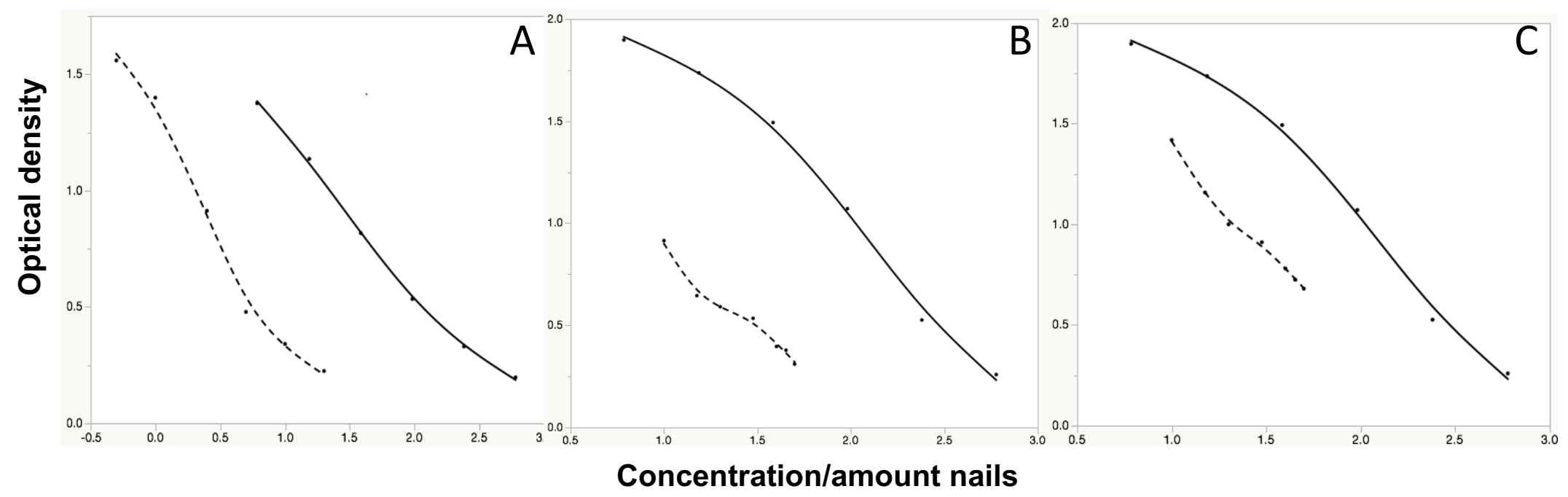

https://mc06.manuscriptcentral.com/cjpp-pubs 


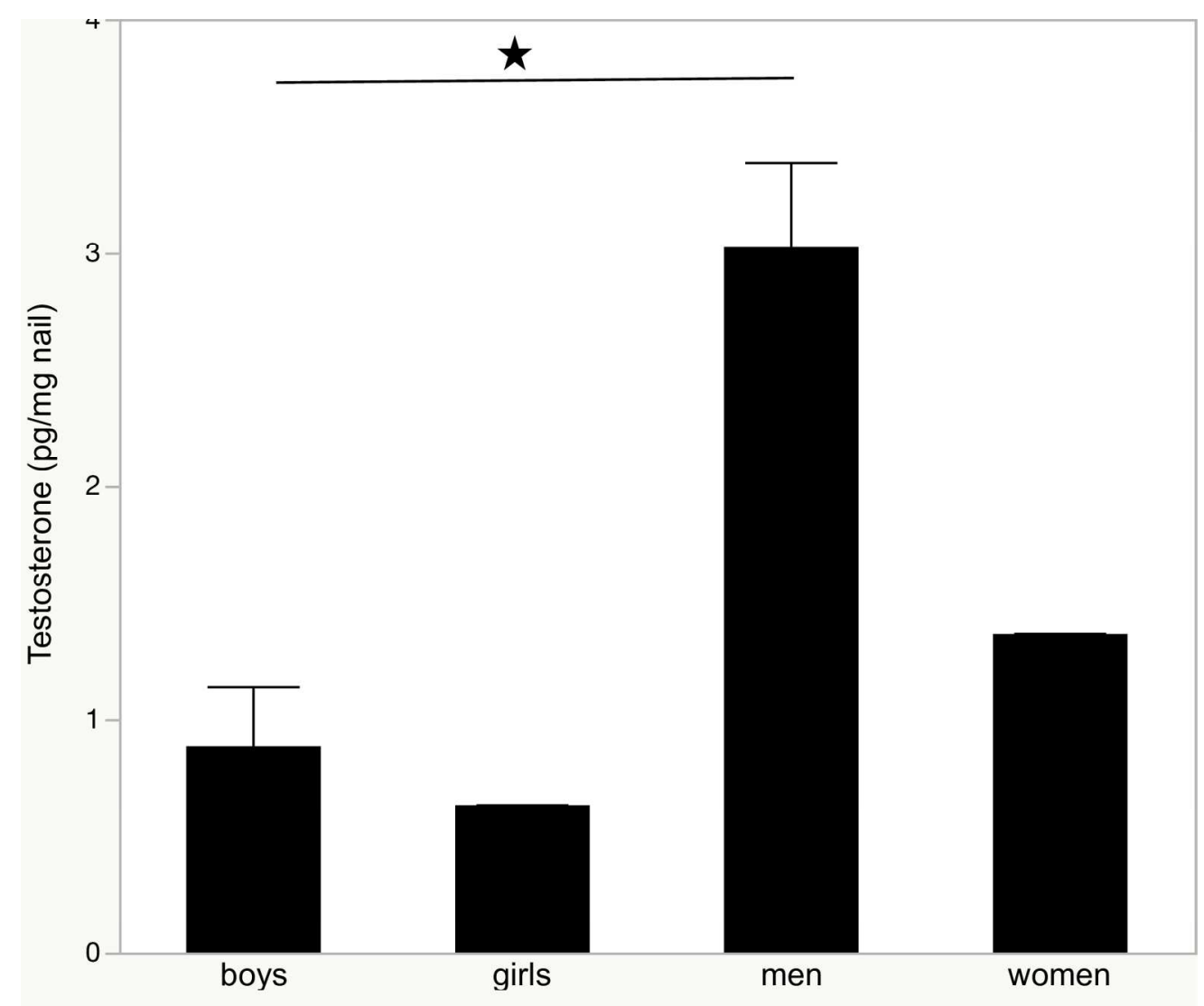

Figure 2

$166 \times 143 \mathrm{~mm}(300 \times 300 \mathrm{DPI})$ 


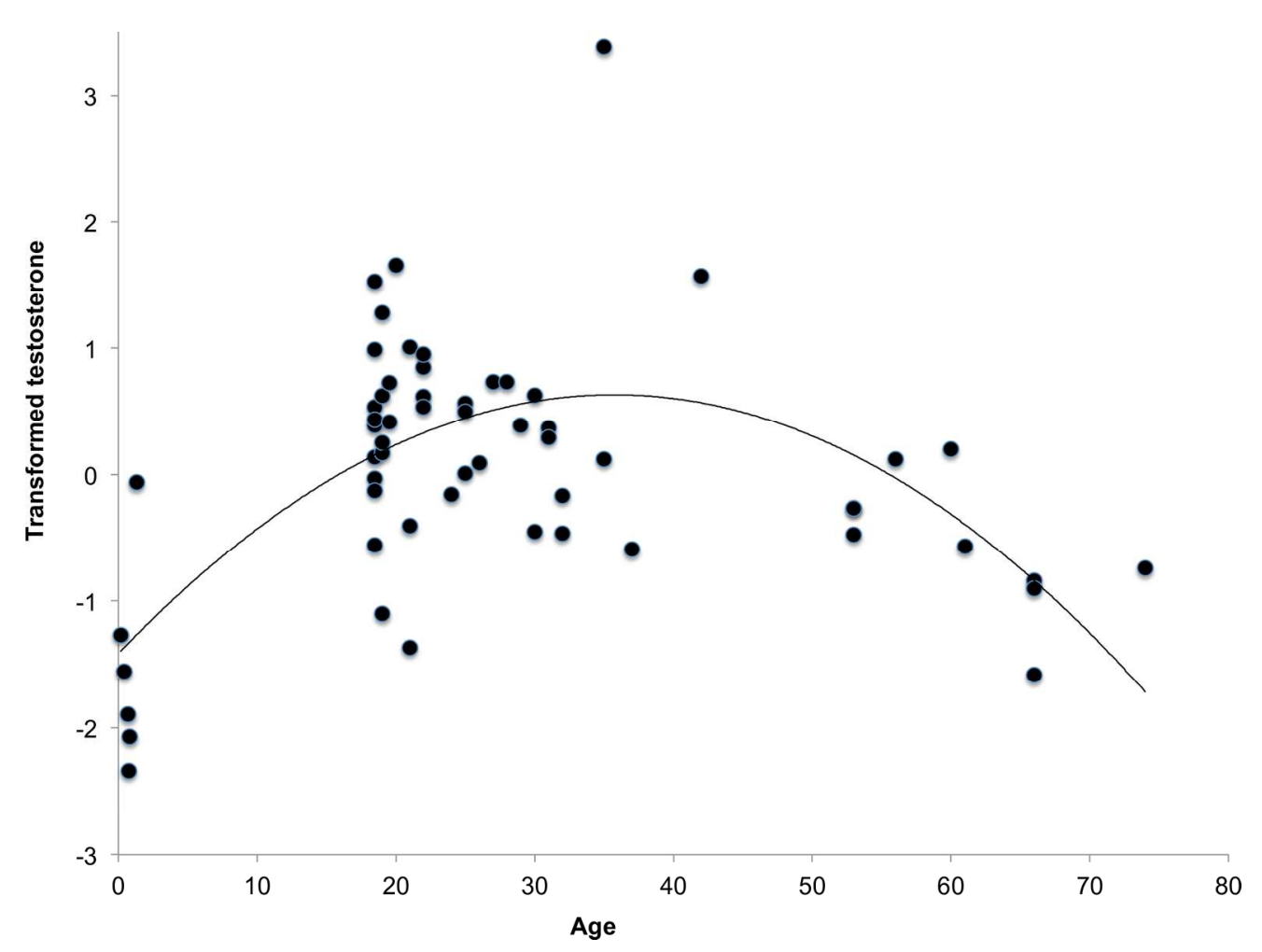

Figure 3

$190 \times 142 \mathrm{~mm}(300 \times 300 \mathrm{DPI})$ 